Check for updates

Cite this: RSC Adv., 2019, 9, 12496

\title{
Fabrication of polyaniline-graphene/polystyrene nanocomposites for flexible gas sensors
}

\author{
Jolly Bhadra, ${ }^{a}$ Anton Popelka, ${ }^{a}$ Asma Abdulkareem, ${ }^{a}$ Zubair Ahmad, ${ }^{a}$ Farid Touati $^{\text {b }}$ \\ and Noora Al-Thani (iD *a
}

This research work presents the fabrication of polyaniline (PANI) and graphene-polyaniline (graphenePANI) nanocomposite-coated polystyrene (PS) nanofibre mats, as well as their application in flexible and highly sensitive gas sensors. The surface morphology of the flexible films is investigated using a number of techniques. The profilometry studies confirmed that the electrospun fibres are evenly distributed over a large surface area and there was no visible difference between coated and uncoated fibres. The SEM morphology studies revealed that a nanocomposite consisting of $10 \mathrm{~nm}$ PANI nanofibres and graphene forms a uniform coating around $3 \mu \mathrm{m}$ diameter PS fiber. AFM showed differences in the 3D surface topography between plain PS nanofibres and coated ones, which showed an increased roughness. Moreover, conductive AFM has indicated an increase in the electrical current distribution from picoamperes to nanoamperes of the PS samples coated with PANI and graphene-PANI because of the applied voltage to the AFM tip that contacted the sample surface. The chemical properties of all the samples are analysed by Fourier transform infrared spectroscopy (FTIR) and X-ray powder diffraction $(X R D)$, which revealed the presence of chemical interactions between the nanocomposites and the polymeric backbones. The TGA study indicated that graphene-PANI coated fibres have the highest thermal stability compared to the pure fibres. The addition of the nanocomposite layer to the PS fibre significantly increased the electrical conductivity. Therefore, nanocomposite-coated flexible membranes are used to fabricate carbon dioxide gas sensors (sensing range: 20-100 ppm). Due to the higher surface area of the nanocomposite coated fibre the availability of adsorption area is also higher, which leads to an increase in sensitivity to carbon dioxide gas. The sensitivity increases with the increase in gas concentration. The average response time of the sensor is calculated to be 65 seconds, with good and uniform repeatability.

Received 4th February 2019 Accepted 13th April 2019

DOI: 10.1039/c9ra00936a

rsc.li/rsc-advances materials, ${ }^{\mathbf{8} 9}$ and organic/inorganic composites ${ }^{\mathbf{1 0 , 1 1}}$ have been used as sensing materials, which have different sensing mechanisms and principles. However, polymer-based gas sensors have an advantage of inherent flexibility.

The performance of a chemical gas sensor is assessed based on parameters such as sensitivity, selectivity, time response, stability, durability, reproducibility, and reversibility, which depend on the properties of the sensing material. ${ }^{\mathbf{1 2}-14}$ The sensitivity is directly affected by the specific surface area of the sensing materials, and a high specific surface area results in higher sensitivity. ${ }^{\mathbf{1 5 , 1 6}}$ This motivates researchers to look for different techniques to increase the specific surface area of sensing materials. The most popular technique is modifying the materials to have nanoscale structure. The nanostructured form of any materials has an advantage of a very large specific surface area. ${ }^{17-19}$ In the last few decades, a number of groups have adopted different chemical and physical methods to obtain different types of nanoparticles. ${ }^{20,21}$ Electrospinning is one of the most robust and reliable methods to prepare polymer nanofibres. $^{22}$
${ }^{a}$ Center for Advanced Materials, Qatar University, P. O. Box 2713, Doha, Qatar. E-mail: n.al-thani@qu.edu.qa

${ }^{b}$ Department of Electrical Engineering, College of Engineering, Qatar University, Doha 2713, Qatar 
The flexibility of polymers makes them very useful. However, one or more conducting material needs to be incorporated to use polymer-based materials in electronics as an electrontransporting material. Examples are conducting polymers, carbon-based materials, graphene, CNTs, and metal oxides. Polymer composite-based gas sensors are one of the most widely studied types of gas and vapour sensors because of their unique responses to each analyte gas. A composite-based gas sensor that consists of polymers and carbon particles shows a change in electrical resistivity when exposed to an analyte gas. Several research groups have observed highly responsive and sensitive polymer-based sensors for the detection of volatile organic compounds. ${ }^{\mathbf{2 0 - 2 2}}$

Polyaniline (PANI) is an intrinsically gas-sensitive conducting polymer. PANI is responsive to environmental pollutants such as $\mathrm{NH}_{3}, \mathrm{CO}, \mathrm{SO}_{2}$, and other greenhouse gases, which is highly dependent on the processing techniques used. Many researchers have used various nano-manufacturing techniques to make PANI-based sensors and evaluated their quality and stability. Other additional advantages of PANI are environmentally friendliness and stability, a simple synthesis method, simple doping-dedoping chemistry, and low cost. ${ }^{23-25}$

Graphene is a commonly used carbon-based single-layered 2D material ${ }^{26-28}$ that has high specific surface area and an extraordinary room-temperature electron mobility of 20000 $\mathrm{cm}^{2} \mathrm{~V}^{-1} \mathrm{~s}^{-1}$. It has a resistivity of $10^{-6} \Omega \mathrm{cm}$, and its carrier density is $10^{12} \mathrm{~cm}^{-2}$. Its electrical, mechanical, and chemical properties are very useful for gas and vapour sensing. ${ }^{29,30}$

Graphene-PANI composites have been successfully investigated by many researchers. However, in most of these cases, binding materials are used during the preparation process, which interfere with the electrical conductivity and complicate the system..$^{31}$ In situ polymerization and solution mixing are two other methods commonly used to prepare PANI graphene nanocomposites. In in situ polymerization, chemical polymerization is done to convert aniline to polyaniline in a medium containing graphene. Solution mixing is a simple method that is done under constant stirring or ultrasonication to make PANI-graphene composites. ${ }^{32,33}$

We have used an in situ electro-polymerization method to coat PANI and graphene-PANI on an electrospun polystyrene (PS) nanofibre film functionalized by using plasma treatment. The flexible nanocomposite-coated PS membranes are characterized by different techniques for morphological, chemical, thermal, and electrical analyses. The performance of nanocomposite-based gas sensors are also analysed in detail.

\section{Materials and experimental methods}

\subsection{Materials}

Aniline $\left(\mathrm{C}_{6} \mathrm{H}_{2} \mathrm{NH}_{2}, \mathrm{MW}=93.13,99.5 \%\right), \mathrm{N}, \mathrm{N}$-dimethylformamide (DMF) (linear formula $\mathrm{HCON}\left(\mathrm{CH}_{3}\right)_{2},>99.8 \%$, molecular weight (MW): $73.09 \mathrm{~g} \mathrm{~mol}^{-1}, d=\sim 0.944 \mathrm{~g} \mathrm{ml}^{-1}$ (lit.)), polystyrene (linear formula $\left[\mathrm{CH}_{2} \mathrm{CH}\left(\mathrm{C}_{6} \mathrm{H}_{5}\right)\right] n$, average MW: $\sim 280.000 \mathrm{~g} \mathrm{~mol}^{-1}, T_{\mathrm{g}}=100{ }^{\circ} \mathrm{C}, d=1.047 \mathrm{~g} \mathrm{ml}^{-1}$ at $25{ }^{\circ} \mathrm{C}$ ), and graphite (empirical formula (Hill Notation) C, MW: $12.01 \mathrm{~g}$ $\mathrm{mol}^{-1}$, powder, $<20 \mu \mathrm{m}$, synthetic, $\mathrm{mp}=3652-3697^{\circ} \mathrm{C}$ (lit.)) are purchased from Sigma Aldrich Co., Ltd, USA. Hydrochloric acid 37\% (HCL, MW: 36.46, $d=1.18 \mathrm{~g} \mathrm{~cm}^{-3}\left(20{ }^{\circ} \mathrm{C}\right)$ ), ammonium peroxodisulphate (APS) $\left(\left(\mathrm{NH}_{4}\right){ }_{2} \mathrm{~S}_{2} \mathrm{O}_{8}, \mathrm{MW}: 228.2 \mathrm{~g} \mathrm{~mol}^{-1}, d=\right.$ $1.98 \mathrm{~g} \mathrm{~cm}^{-3}\left(20{ }^{\circ} \mathrm{C}\right)$ ), sodium nitrate $\left(\mathrm{NaNO}_{3}, \mathrm{MW}: 84.99 \mathrm{~g}\right.$ $\left.\mathrm{mol}^{-1}, d=2.26 \mathrm{~g} \mathrm{~cm}^{-3}\left(20^{\circ} \mathrm{C}\right)\right)$, and sulphuric acid $95 \%\left(\mathrm{H}_{2} \mathrm{SO}_{4}\right.$, MW: $\left.98.08 \mathrm{~g} \mathrm{~mol}^{-1}, d=1.84 \mathrm{~g} \mathrm{~cm}^{-3}\left(20^{\circ} \mathrm{C}\right)\right)$ are purchased VWR CHEMICALS Analwr Normapur. Potassium permanganate $\left(\mathrm{KMnO}_{4},>99.0 \%, d=158.04 \mathrm{~g} \mathrm{~mol}^{-1}\right)$ and hydrogen peroxide $30 \%\left(\mathrm{H}_{2} \mathrm{O}_{2}, 29.0 \%\right.$ specific gravity) are purchased from $\mathrm{BDH}$ Laboratory Supplies Pools, BH15 TD, England. All the chemicals are used without further treatment.

\subsection{Experimental methods}

\subsubsection{Sample preparation}

2.2.1.1 Electrospinning. PS nanofibre membrane is prepared by electrospinning at room temperature. PS solution is prepared by dissolving PS at $20 \% \mathrm{w} / \mathrm{v}$ in DMF, and then the solution is stirred continuously in an oil bath while maintaining $60{ }^{\circ} \mathrm{C}$ for $24 \mathrm{~h}$ prior to electrospinning. After that, $5 \mathrm{ml}$ of the solution is loaded in a $10 \mathrm{ml}$ syringe with a stainless-steel needle with an inner diameter of $0.23 \mathrm{~mm}$. Electrospinning is performed using a setup with a horizontal configuration. The working conditions are as follows: a $15 \mathrm{~cm}$ distance from the needle to the collector, a flux of $1.5 \mathrm{ml} \mathrm{h}^{-1}$ provided by a syringe pump, and a voltage of $15 \mathrm{KV}$ applied to the needle to yield nanofibres. The fibres are collected on a rotated collector covered with aluminium foil and then dried at room temperature for $24 \mathrm{~h}$. The PS mat is sandwiched between two pieces of aluminium foil and then pressed using a weight of $250 \mathrm{~g}$ for $24 \mathrm{~h}$ to make the PS membrane stable and easy to handle.

2.2.1.2 Plasma treatment. The surface of the PS membrane in aluminium foil is treated under dynamic conditions at atmospheric pressure using a radio frequency plasma force system (Venus 75-HF, PlasmaEtch, USA). Plasma treatment is performed for $30 \mathrm{~s}$ in air. Both sides of the membrane are treated with a high-frequency sinusoidal voltage $(13.56 \mathrm{MHz})$. The equipment allows the generation of macroscopically homogenous low-temperature plasma for uniform surface treatment.

2.2.1.3 Graphene preparation. Graphene oxide (GO) is prepared by the modified Hummers' method. In this method, $3 \mathrm{~g}$ of graphite powder and $1.5 \mathrm{~g}$ of sodium nitrate are added to $69 \mathrm{ml}$ of concentrated sulphuric acid and stirred vigorously at $0{ }^{\circ} \mathrm{C}$ for $3 \mathrm{~h}$. Then $9 \mathrm{~g}$ of potassium permanganate is slowly added to the mixed solution while maintaining the temperature at $0{ }^{\circ} \mathrm{C}$ in an ice bath. The solution is then removed from the ice bath and stirred at $35{ }^{\circ} \mathrm{C}$ overnight. The solution turns pasty and dark green in colour with effervescence, and $150 \mathrm{ml}$ of cold deionized water is added slowly, which produces a large exothermic reaction $\left(98^{\circ} \mathrm{C}\right)$.

The mixture is maintained at this temperature and stirred for $30 \mathrm{~min}$ until the solution colour becomes brown. Lastly, $10 \mathrm{ml}$ of hydrogen peroxide is added to the mixture, the solution turns bright yellow, the whole suspension is filtered, and the 

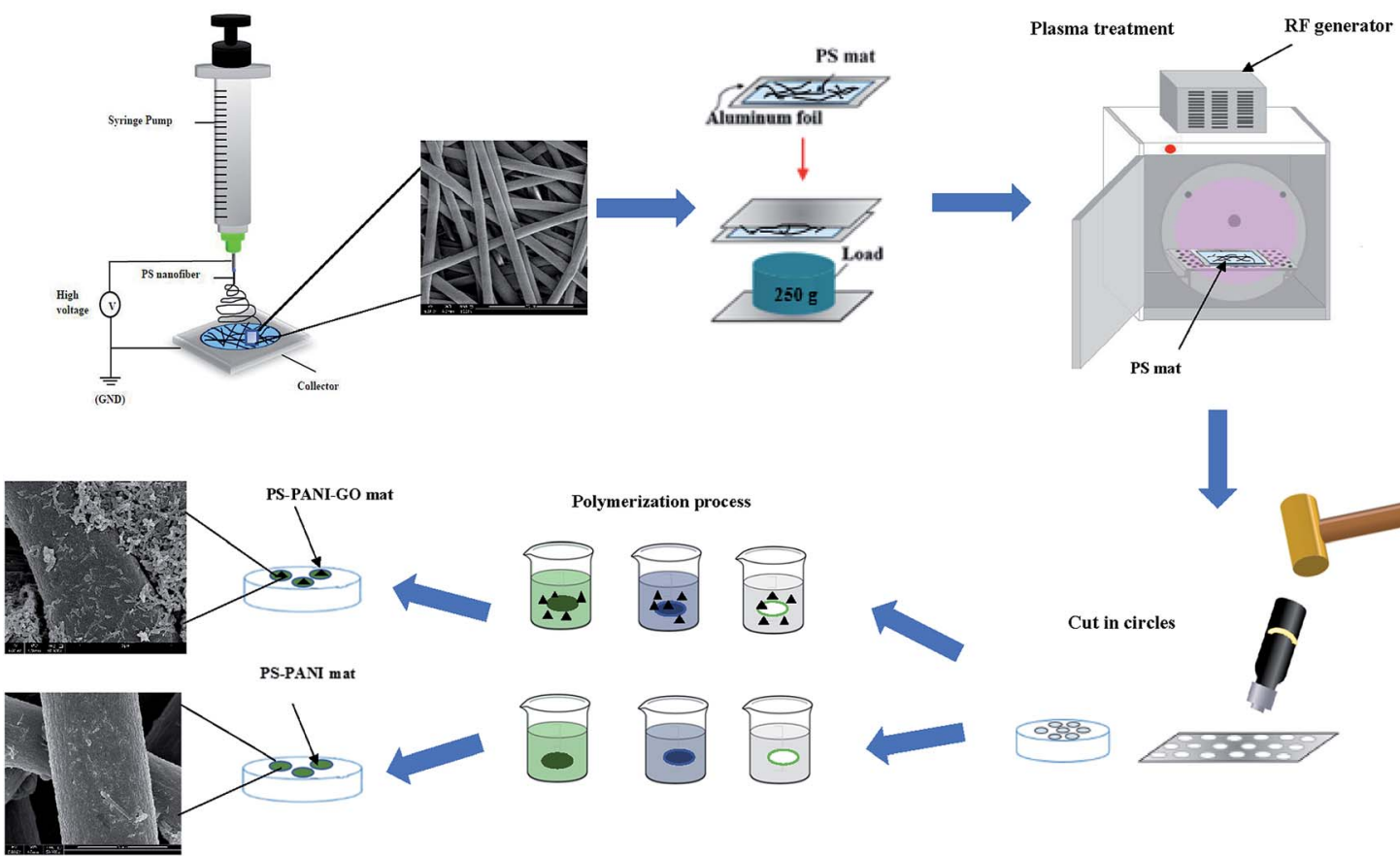

Fig. 1 Preparation steps followed to obtain flexible PANI and PANI, graphene on PS fibre mat.

obtained GO cake filtrate is washed with an excess amount of warm distilled water. Further purification of GO is done to remove any large aggregates, and the GO is sonicated in deionized water for 30 minutes and centrifuged at $1000 \mathrm{rpm}$ for 20 minutes. The supernatant is carefully collected in a Petri dish and allowed to dry at $40{ }^{\circ} \mathrm{C}$ overnight. The GO is reduced to graphene under a hydrogen environment.

2.2.1.4 Polymerization. PANI coating on PS membrane is performed by using chemical polymerization method using aniline in an aqueous acidic medium (PH-4). In this process, $1 \mathrm{M}$ stock solution of HCL and deionized water is prepared. Plasma treated PS membranes are added to the beaker containing HCL stock solution. Then, aniline is added as a monomer into this solution with constant shaking. Ammonium persulphate with a constant molar ratio with $\mathrm{HCl}$ is added dropwise to the aniline over a period of 30 minutes. The solution turns green, indicating the formation of PANI. The reaction is left under constant shaking overnight in an ice bath while maintaining a temperature of $0-5{ }^{\circ} \mathrm{C}$ to obtain a polymerized dark-green precipitate. The PS membranes are removed from the polymerized solution and washed several times with distilled and dried in room temperature for $24 \mathrm{~h}$ before use.

In order to prepare graphene-PANI coated PS membrane we start with graphene in $\mathrm{HCl}$ stock solution and repeated abovementioned steps shown in Fig. 1. Optical images of neat PS and coated membranes and demonstration of flexible membrane are shown in Fig. 2(a and b).

2.2.2 Characterization techniques. Scanning electron microscopy (SEM) is used to study the surface morphology of the electrospun PS nanofibres, as well as the grain size of the PANI, graphene, and graphene-PANI composite (NanoSEM Nova 450). A 3D optical surface metrology system (DCM8 profilometer, Leica, Germany) is used to analyse the surface morphology of the PS electrospun nanofibre membrane and graphene-PANI deposited on the membrane surface. The detector is able to obtain 3D images of relatively large surface areas. The images of these samples are captured using an EPI $50 \times-\mathrm{L}$ objective $(1360 \times 1024$ data points in an area of $350.62 \times$ $263.93 \mu \mathrm{m}^{2}$ ).

Information about the surface morphology structures is obtained from a small surface area $\left(3 \times 3 \mu \mathrm{m}^{2}\right)$ and the electrical characteristics are determined by conductive atomic force

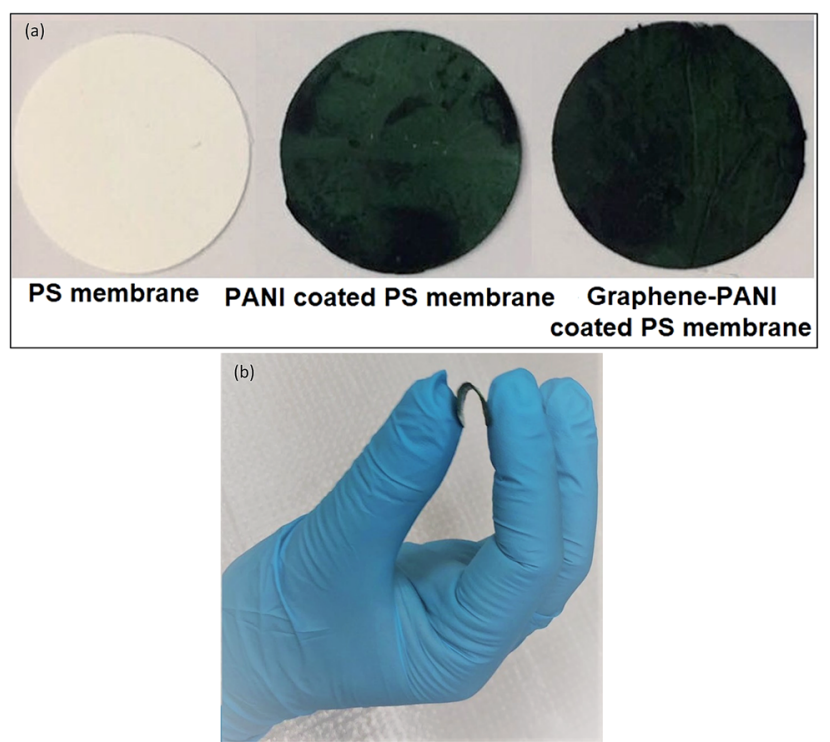

Fig. 2 Optical images of (a) neat PS membrane, PANI coated and graphene-PANI coated PS membrane (b) image of flexible membrane. 


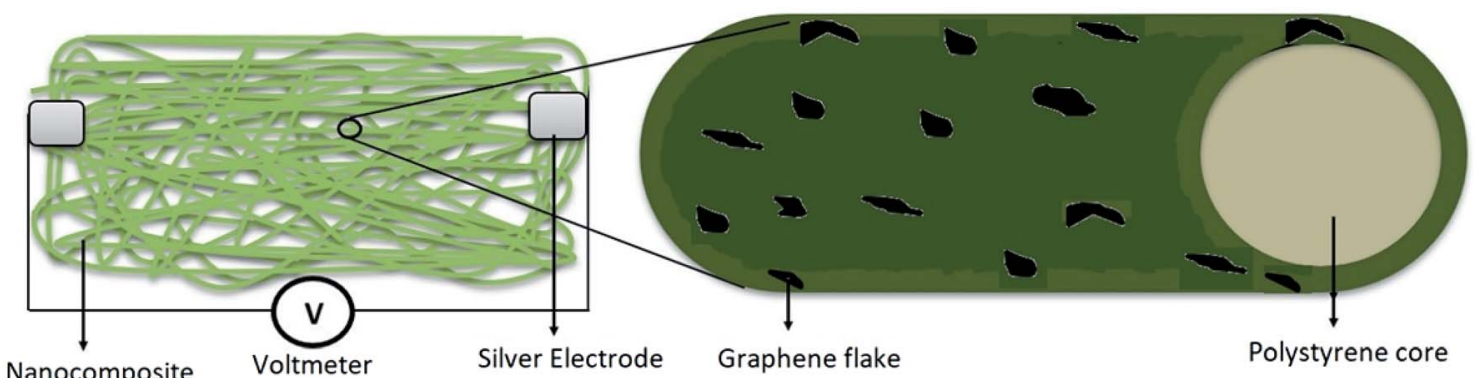

coated Electrospin

PS fibers

Fig. 3 Diagram of the electrical measurement setup.

microscopy (AFM). MFP-3D AFM equipment (Asylum research, USA) with an ORCA accessory is used in these analyses. Measurements are carried out under ambient conditions with the assistance of a silicon probe (Al reflex coated Veeco model OLTESPA, Olympus). The conductive AFM is able to map the topography (height and sensor deflection) and the current distribution simultaneously during the measurement and gave images of the current distribution at a particular bias voltage. The ORCA accessory consists of a cantilever holder that includes a transimpedance amplifier. The amplifier gain is $5 \times$ $10^{8}$ volts per amp, which ensures that a current response in the range of $0.6 \mathrm{pA}$ up to $20 \mathrm{nA}$ can be measured. The current distribution is recorded in response to a voltage applied to the AFM tip while contacting the sample surface.

A Fourier-transform infrared spectrometer (FTIR) (8101 M, Shimadzu) is used to the study the chemical interaction between the polymers. To study the thermal properties of the PANI, thermogravimetric analysis (TGA) and differential scanning calorimetry (DSC) are performed using a PerkinElmer Pyris thermogravimetric analyser and PerkinElmer Precisely Jade differential scanning calorimeter, respectively. The electrical properties are studied by measuring the in-plane $I-V$ characteristics and four-probe conductivity. Fig. 3 shows the scheme used for measuring the electrical properties. A Keithley 2400 source meter is used for the $I-V$ and conductivity measurements of the blends.

\section{Results and discussion}

\subsection{Morphological analysis}

3.1.1 Profilometry. The 3D surface topographies of the fibres are obtained using an optical metrology system. This technique captured images with high resolution in a relatively large surface area $\left(350.62 \times 263.93 \mu \mathrm{m}^{2}\right)$. The surface topography of the fibres is uniform and homogeneous (Fig. 4). The coatings of PANI and graphene-PANI on the PS has not lead to any visible changes, and the fibres are evenly distributed in the prepared layers.
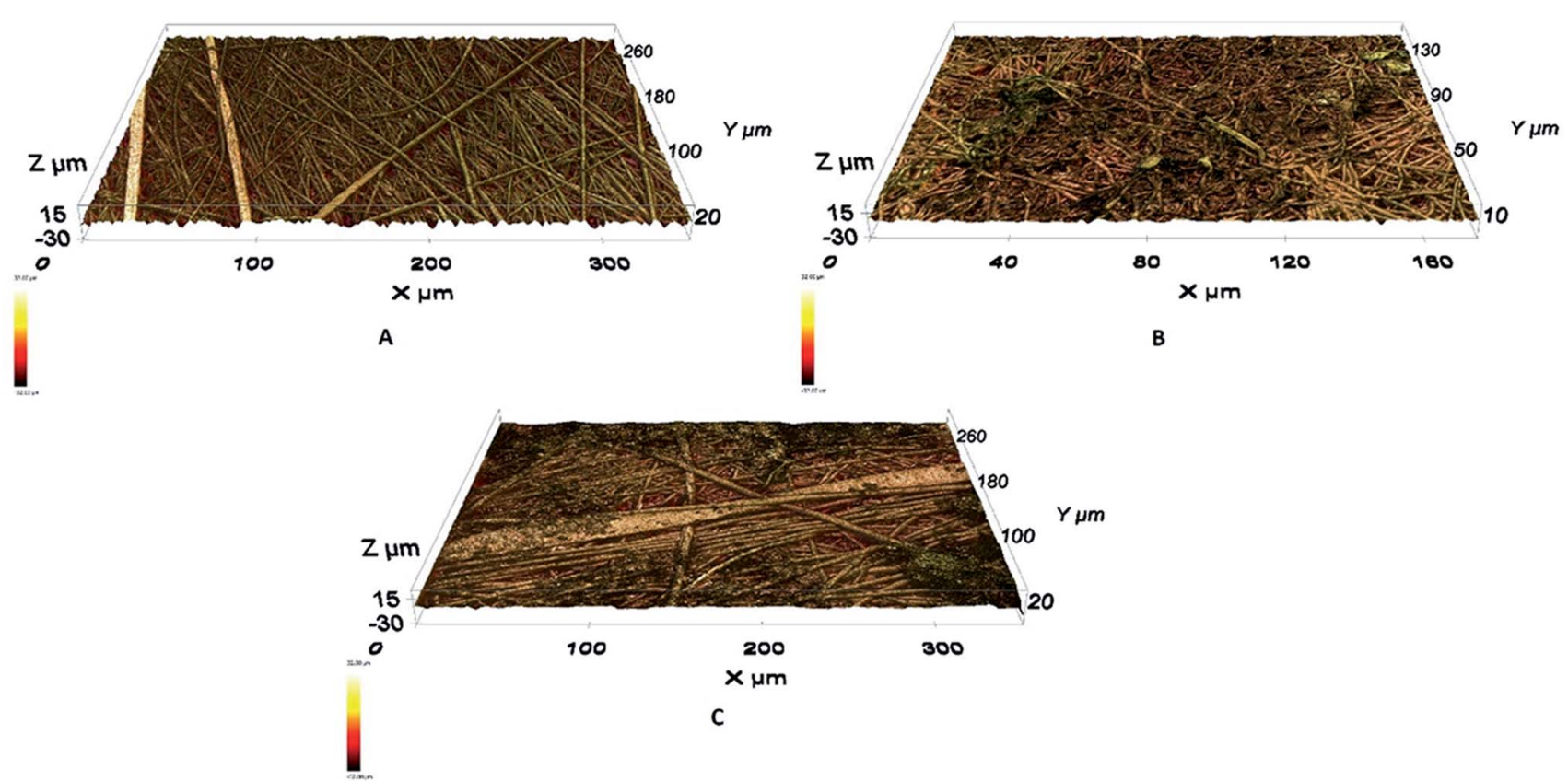

Fig. 4 Optical surface morphology images of prepared layers: (A) PS fibres, (B) PANI coated PS fibres, (C) graphene-PANI coated PS fibres. 
3.1.2 Scanning electron microscopy. The performance of any gas sensor basically depends on the available specific surface for exposure to the analyte gas. In order to determine the available surface area, SEM analysis is done for all the electrospun mats, and the images are shown in Fig. 5. The morphology of the neat electrospun PS fibres is uniform, and the fibres have an average diameter of $3 \mu \mathrm{m}$ (Fig. 5A). The magnified image of a single fibre in Fig. 5B shows a porous surface. SEM images of both the PANI (Fig. 5C, D and $\mathrm{H}$ ) and graphene-PANI (Fig. 5E-G) coated on the PS fibre mats show a uniform coating on the surfaces. However, the graphene-PANI coating may influence the availability of specific area for gas sensor applications. The pure PANI image in Fig. 5H shows fibrous morphology with a diameter of about $10 \mathrm{~nm}$ and length of $100 \mathrm{~nm}$.

3.1.3 Atomic force microscopy. Information about the surface topography of particular electrospun fibres is obtained by conductive AFM with an ORCA accessory in contact mode (Fig. 6). The surface of plain PS fibres showed a relatively smooth and uniform structure without any defects (Fig. 6A). The coating of PS fibres by PANI (Fig. 6B) or graphene-PANI (Fig. 6C) lead to rougher surface structures. AFM is also used to analyse the electrical current distribution in response to the applied voltage to the AFM tip. These images show an increase in the electrical current response from picoamperes to nanoamperes after coating with PS by PANI as a result of the enhanced electrical conductivity. Moreover, the addition of graphene to the PANI coating resulted in a more even distribution of the electrical current in the whole measured surface area due to improvement of the electrically conductive paths.

\subsection{Chemical analysis}

3.2.1 Fourier transform infrared spectroscopy. Fig. 7A shows the FTIR spectra of the neat PS, PANI-coated PS mat, and
graphene-PANI-coated PS mat. We also include the FTIR spectra for pure PANI and graphene in Fig. 7B for comparison. For pure PS, a band is observed at 3081.2 to $3001.11 \mathrm{~cm}^{-1}$ due to $\mathrm{C}-\mathrm{H}$ aromatic tension, at $2923.91 \mathrm{~cm}^{-1}$ and $2850.40 \mathrm{~cm}^{-1}$ because of $\mathrm{CH}_{2}$ asymmetric and symmetric tension, and at $19430.19-1728.23 \mathrm{~cm}^{-1}$ as a result of aromatic ring monosubstitution. The peak at $1452.28 \mathrm{~cm}^{-1}$ is due to deformation of the aromatic ring, and the peak at $1069.65 \mathrm{~cm}^{-1}$ is due to in plane $\mathrm{C}-\mathrm{H}$ flexion.

All the signature peaks of PS are seen in the IR spectra of PANI-coated and graphene-PANI-coated PS mats. However, there are differences in the intensity. Pure PANI has a strong IR absorption peak at $3430 \mathrm{~cm}^{-1}$. Both the PANI-coated FTIR spectra show peaks at approximately 2947-2926 $\mathrm{cm}^{-1}$ and 2857-2861 $\mathrm{cm}^{-1}$ because of $\mathrm{NH}_{2}, \mathrm{C}-\mathrm{H}$, and $\mathrm{C}-\mathrm{H}_{2}$ stretching, respectively. Another absorption band at $1600-1560 \mathrm{~cm}^{-1}$ is assigned to benzenoid-form $\mathrm{C}-\mathrm{C}$ ring vibration. The vibrational stretching of $N$-benzenoid ring has been seen at 1496$1493 \mathrm{~cm}^{-1}$. The band at $1450-1461 \mathrm{~cm}^{-1}$ is assigned to the $\mathrm{C}-\mathrm{N}$ stretching of the quinoid ring, which is caused by the protonation of the PANI matrix by the dopants.

The band at $1400-1406 \mathrm{~cm}^{-1}$ is assigned to the out-of-plane $\mathrm{CH}$ bending of the $\mathrm{CH}_{3}$ groups, and the band at 1299$1305 \mathrm{~cm}^{-1}$ is assigned to the out-of-plane $\mathrm{NH}$ bending. The FTIR spectrum of pure graphene has no strong transmittance peak. The absence of graphite and graphene oxide peaks is a strong indication that graphene sheets ware obtained. However, both graphene and graphene-PANI-coated PS have a peak at $1620 \mathrm{~cm}^{-1}$, which can be attributed to aromatic carbon double bonds.

3.2.2 X-ray diffractometry results. XRD spectroscopy is used to investigate the effect of the coatings on the structure of the PS nanofibres. The spectrum of pure PS has two
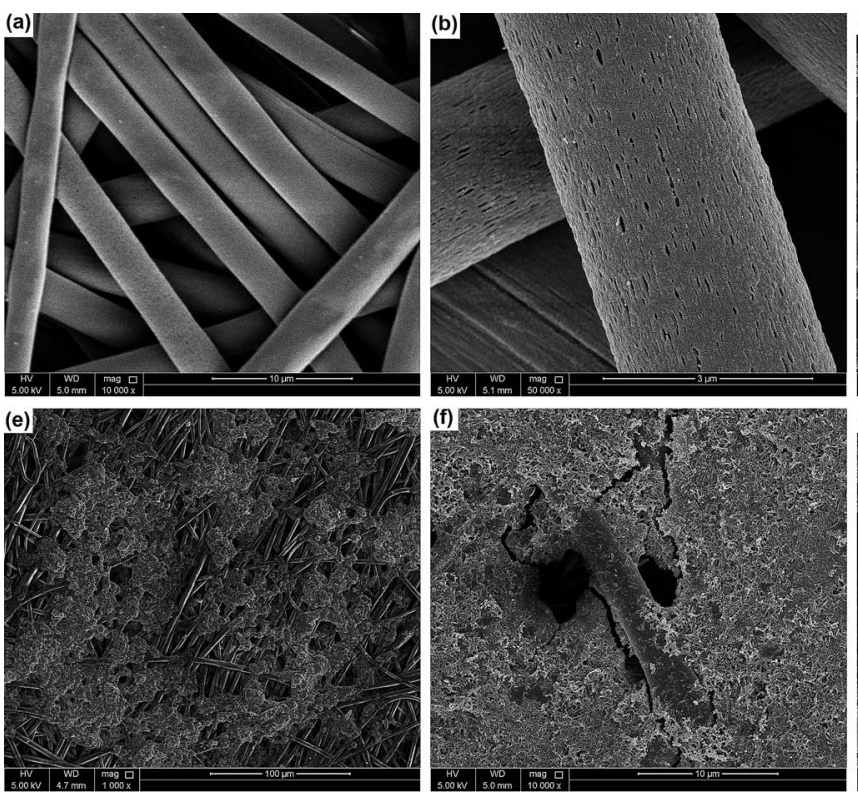
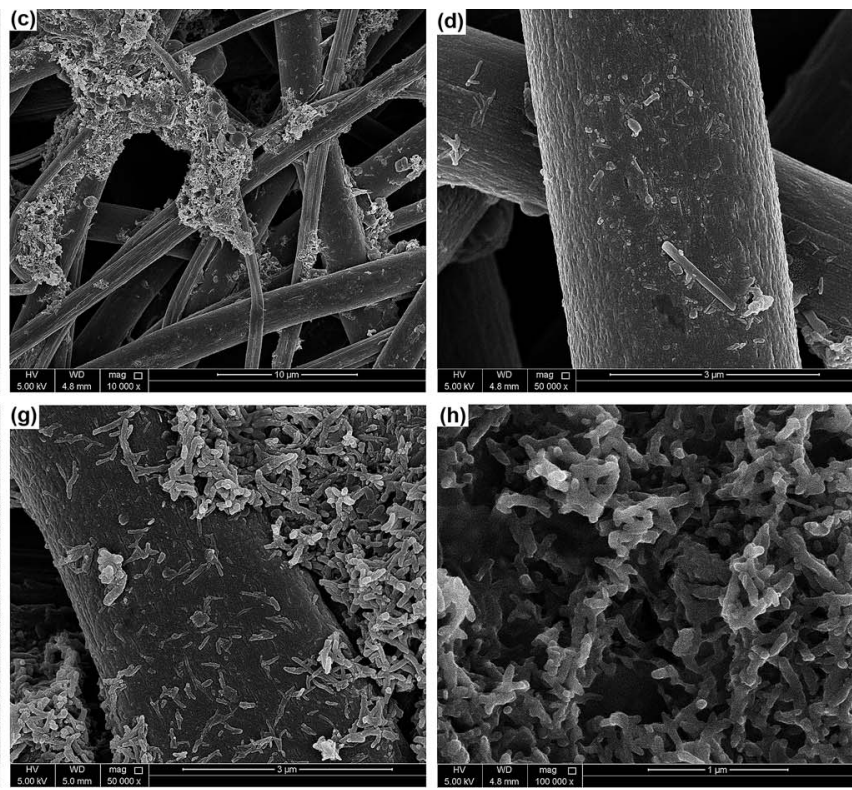

Fig. 5 SEM images of (A) electrospun PS fibre mat, (B) magnified image of PS fibre, (C) PANI-coated PS fibres, (D) magnified image of PANIcoated PS fibre, (E and F) graphene-PANI-coated PS fibres at low magnification, (G) magnified image of graphene-PANI-coated PS fibre, and (H) fibrous PANI nanoparticles. 

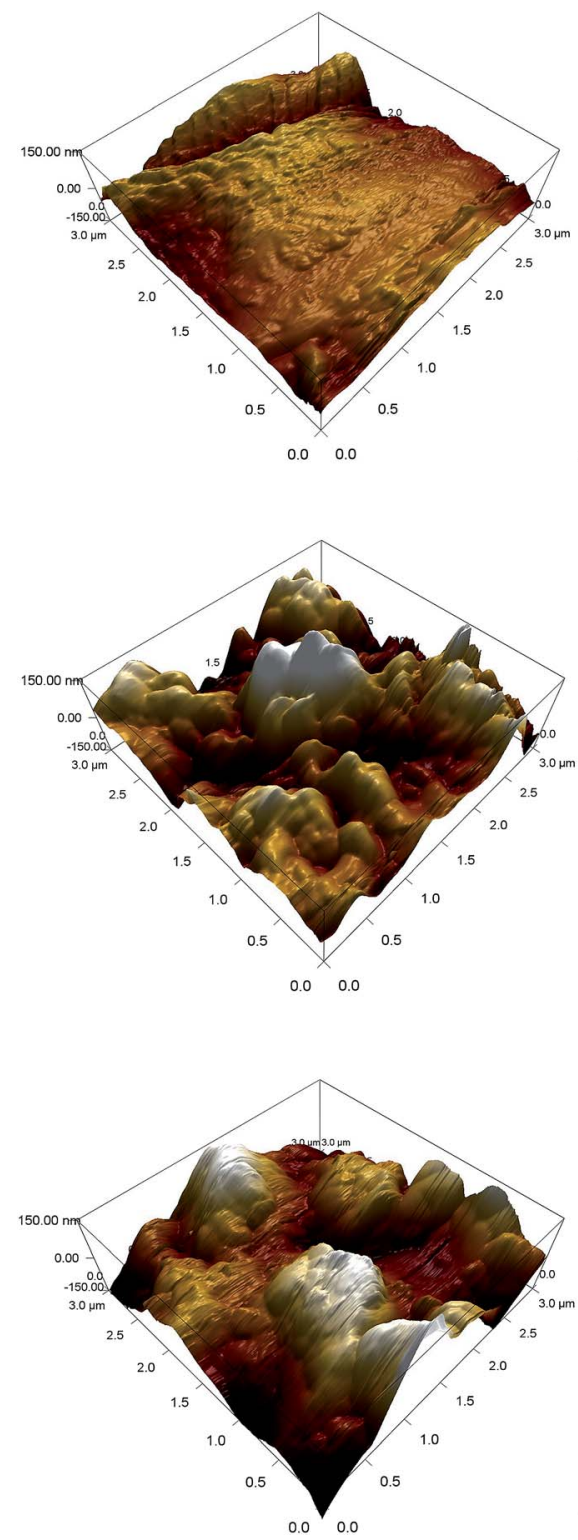
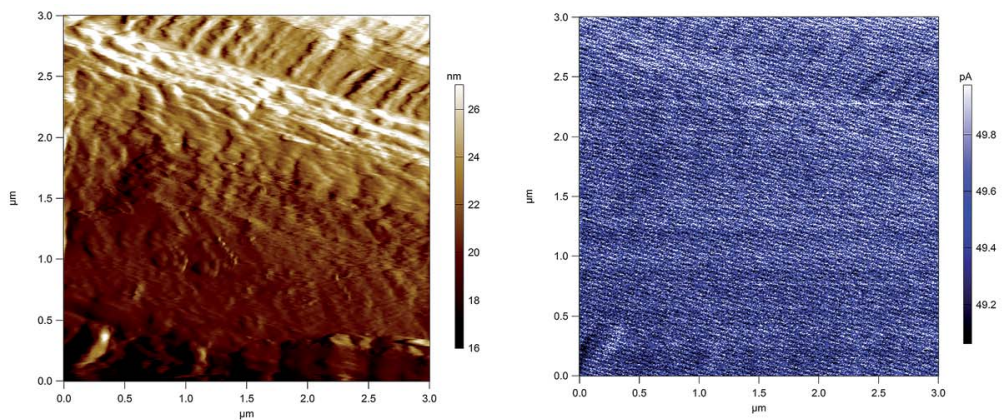

A
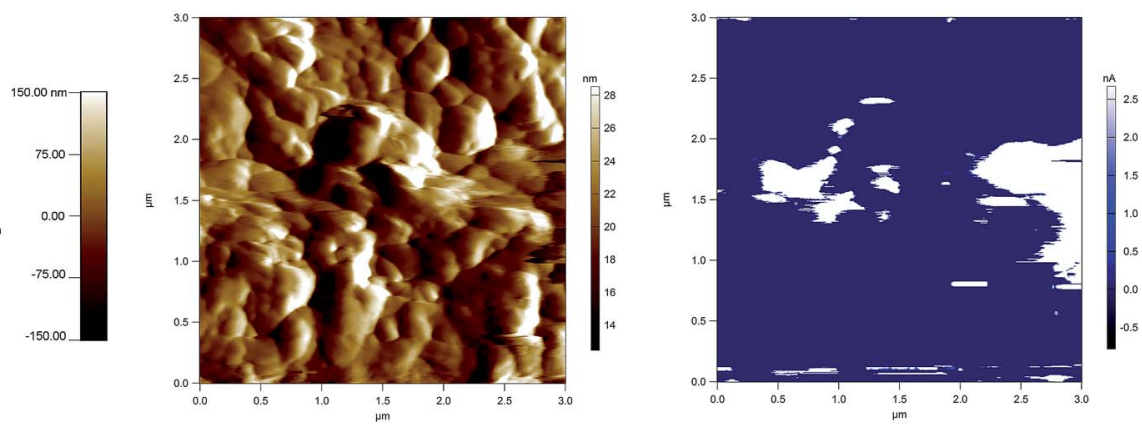

B
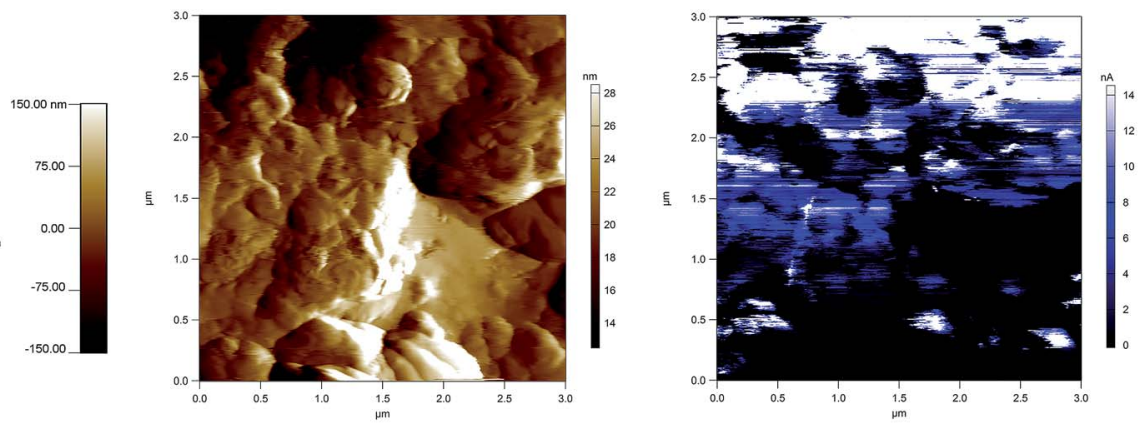

C

Fig. 6 AFM images (3D-height, deflection, and current distribution) of (A) PS nanofibre, (B) PANI-coated PS fibres, (C) graphene-PANI-coated PS fibres.

characteristic amorphous peaks at $2 \theta$ values of 10 and 20 . The XRD images of composite systems in Fig. 8a have broad characteristic amorphous peaks of PS. There is also a number of sharp peaks of varying heights, which are due to the nanocrystalline form of the PANI and graphene-PANI composites during in situ polymerization and deposition on the surface of the PS nanofibres. However, the XRD peaks of pure PANI (Fig. 8b) have diffraction angles of 19.311 and $25.721^{\circ}$, which indicate low crystallinity because of the repeated benzenoid and quinoid rings in the PANI backbone. The XRD result of graphene shows a small amorphous hump, which is suppressed by the high-intensity composite peaks of the polymers.

\subsection{Thermal analysis}

3.3.1 Differential scanning calorimetry. DSC analysis is done to elucidate the interactions among the constituents in the systems, which are responsible for the structural changes, phase transitions, and electrical conductivity of the composites, as shown in Fig. 9(a and b). For precise thermal analysis, three-step scanning is performed with heating from $30{ }^{\circ} \mathrm{C}$ to $200{ }^{\circ} \mathrm{C}$, cooling from $200{ }^{\circ} \mathrm{C}$ to $30{ }^{\circ} \mathrm{C}$, and heating from $30{ }^{\circ} \mathrm{C}$ to $200{ }^{\circ} \mathrm{C}$ at a rate of $10^{\circ} \mathrm{C} \mathrm{min}^{-1}$ under $\mathrm{N}_{2}$ atmosphere. It is well known that the glass transition temperature is appears as a sudden change in slope in the DSC curve or with an endothermic dip at lower temperature. ${ }^{34}$ Glass transition temperature $\left(T_{\mathrm{g}}\right)$ of PS observed in all the samples at around $104{ }^{\circ} \mathrm{C}$. No any melting or crystallization peak is observed in the DSC thermogram of pure PS, however the coated samples have one small melting and one crystallization peaks. The first heating cycle leads to the evaporation of water and other solvents used during the preparation of the PS fibres, PANI, and graphene. Both the second cooling 
$(\mathrm{A})$

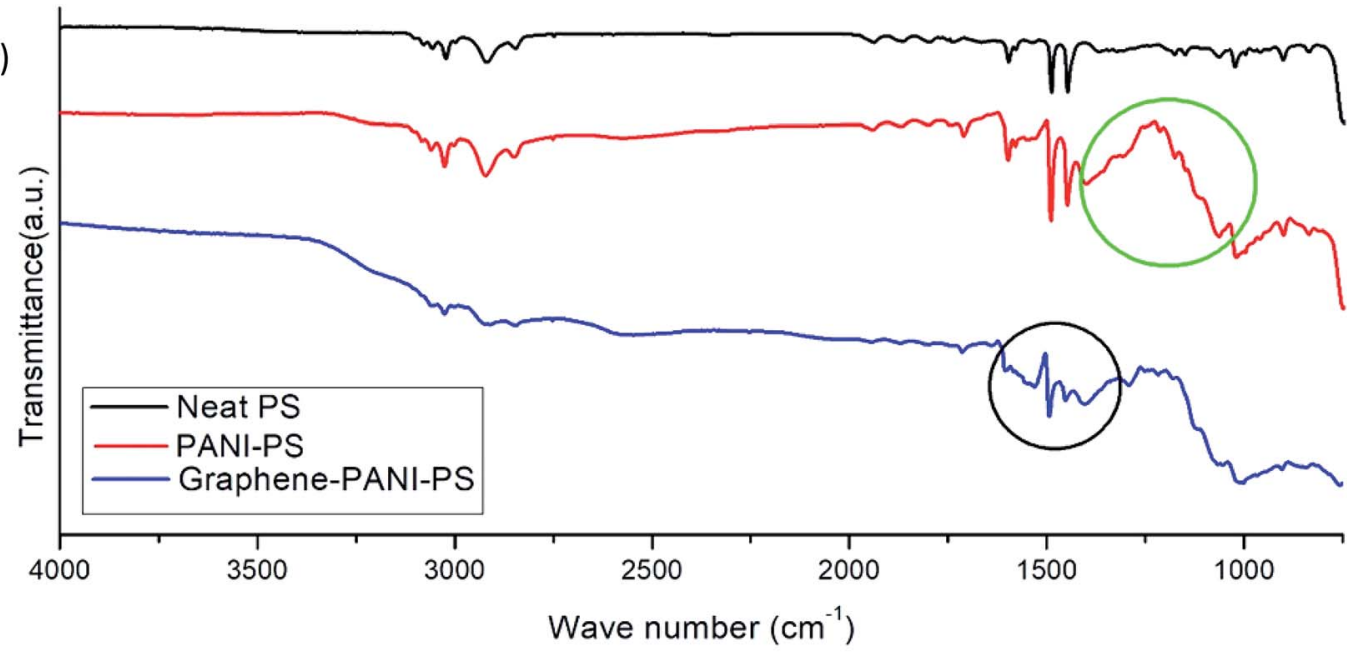

(B)

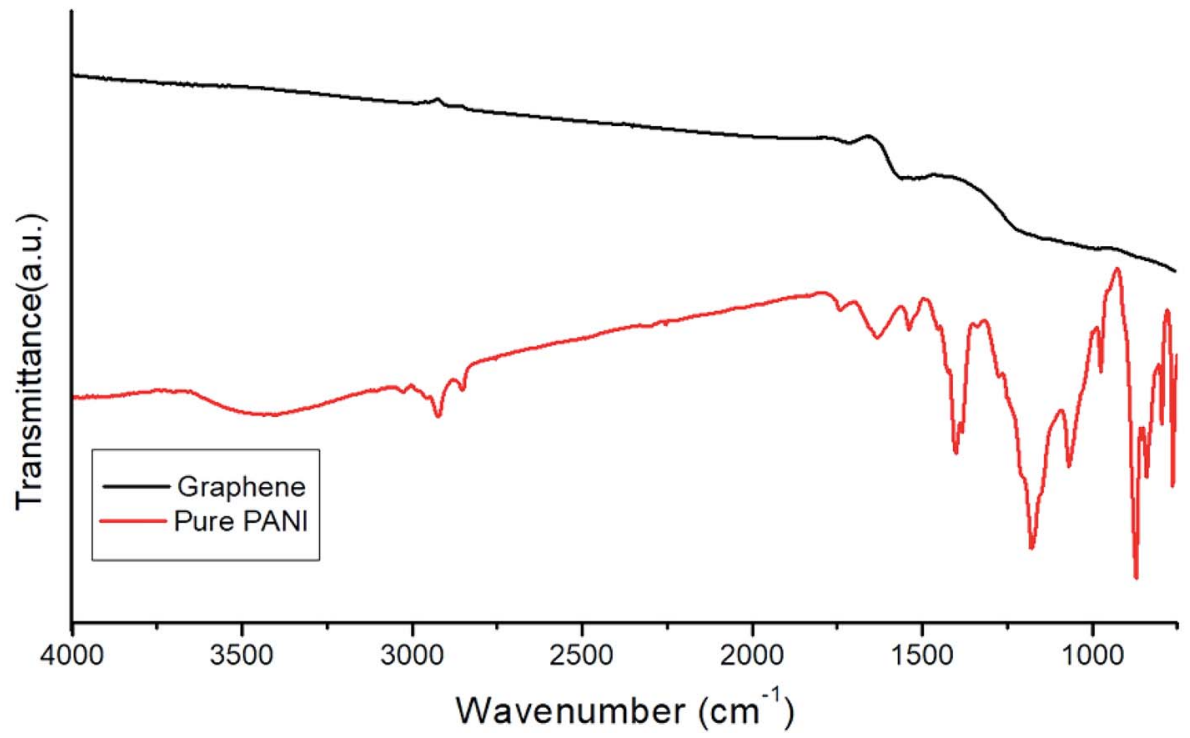

Fig. 7 (A) FTIR images of PS fibres, PANI-coated PS fibres, and graphene-PANI-coated PS fibres. (B) FTIR images of graphene and pure PANI.

cycle and the third heating cycle have a small crystallization peak at $134{ }^{\circ} \mathrm{C}$ and melting peak at $137^{\circ} \mathrm{C}$. The appearance of these peaks may be due to melting and crystallization of a compound that formed due to the interaction of aniline and PS during the polymerization process of aniline. Because similar peaks appear in both the PANI and graphene-PANI-

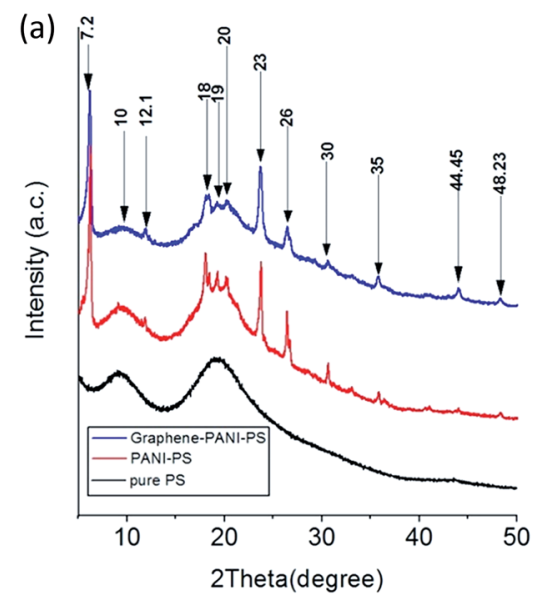

(b)

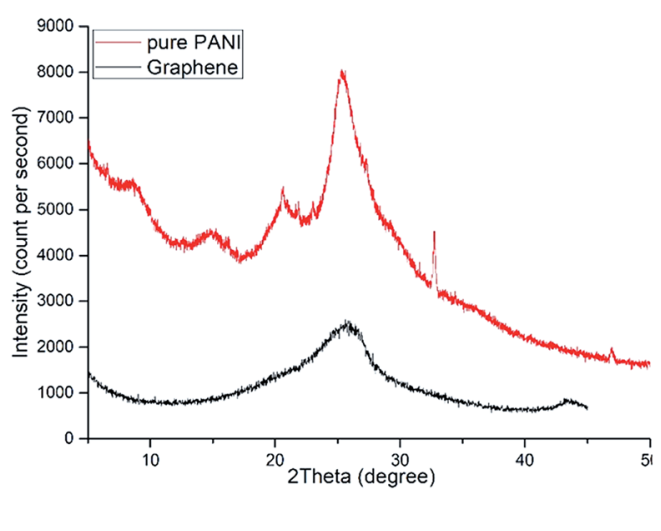

Fig. 8 (a) XRD images of PS fibres, PANI-coated PS fibres, and graphene-PANI-coated PS fibres. (b) XRD images of graphene and pure PANI. 
(a)
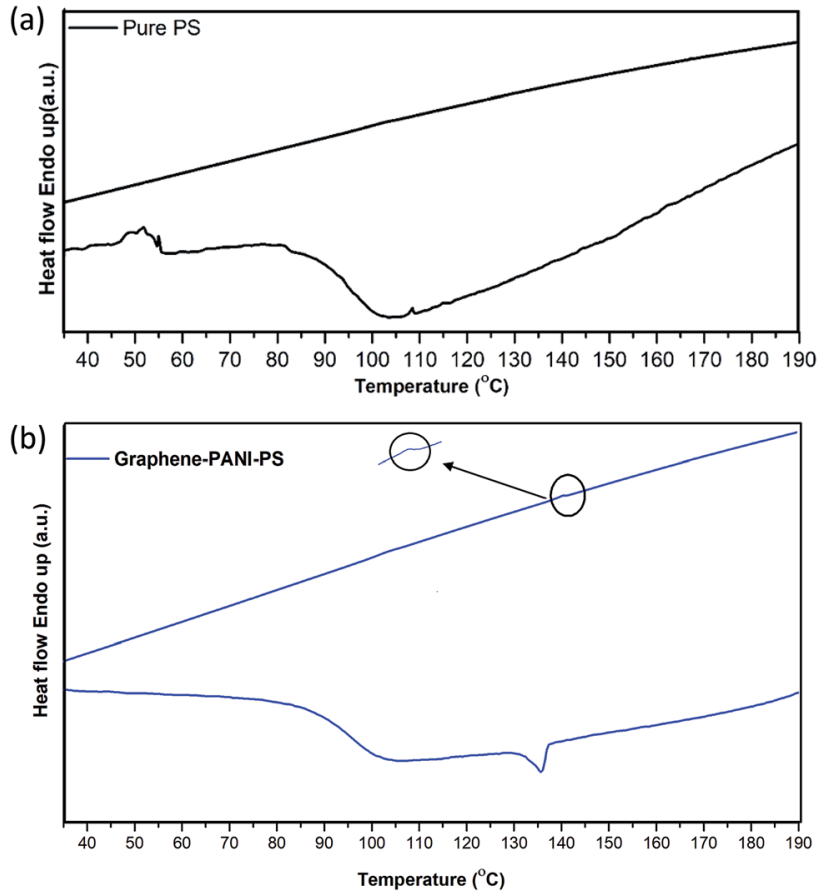

(c)

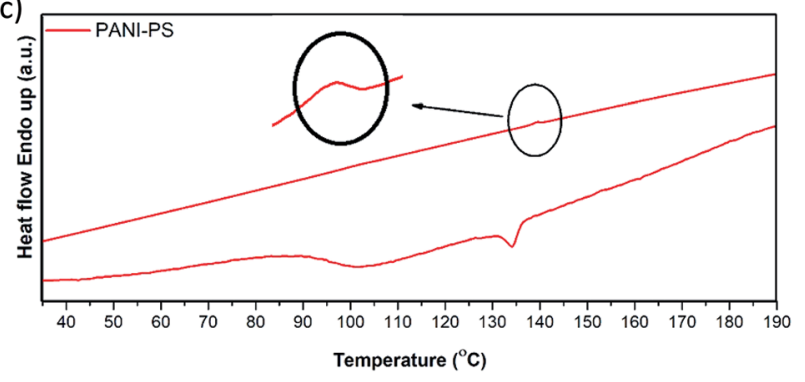

Fig. 9 DSC thermogram for PS fibres, PANI-coated PS fibres, and graphene-PANI-coated PS fibres while heating from $30^{\circ} \mathrm{C}$ to $200^{\circ} \mathrm{C}$, while cooling from $200{ }^{\circ} \mathrm{C}$ to $30^{\circ} \mathrm{C}$.

coated samples, they can be due to the presence of PANI in the sample and addition of graphene has not brought any change in the thermal properties.

3.3.2 Thermal gravimetric analysis. TGA is done under a heating rate of $10 \mathrm{~min}^{-1}$ from room temperature to $600{ }^{\circ} \mathrm{C}$, as shown in Fig. 10. The results reveal that pure PS has $100 \%$ degradation in one step around $418{ }^{\circ} \mathrm{C}$, whereas the coated samples have multiple degradation steps. The thermal degradation of PANI coated PS fibres starts soon with the rise of temperature above $30{ }^{\circ} \mathrm{C}$. The $1^{\text {st }}$ step of weight loss is seen within $100-120^{\circ} \mathrm{C}$, is due to evaporation of water molecules and solvents used during preparation..$^{35}$ The $2^{\text {nd }}$ weight loss step lies between $180-220^{\circ} \mathrm{C}$, is related with the sample deprotonation and loss of dopants from deeper sites in the material. ${ }^{34,35}$ In the third step, of weight loss profiles observed between $250-400{ }^{\circ} \mathrm{C}$, is due to degradation of oligomers and unreacted polymers..$^{35,36}$ Above $435{ }^{\circ} \mathrm{C}$, the weight loss corresponds to the complete decomposition of the polymer. Based on the final degradation temperature, the PANI-graphene-coated PS fibre has the highest thermal stability. After decomposition and degradation of

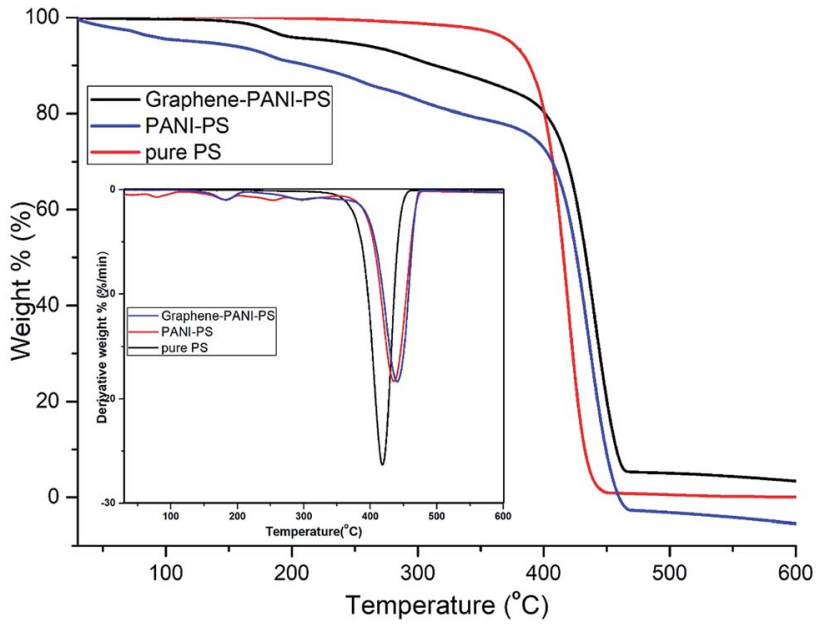

Fig. 10 TGA thermograms for PS fibres, PANI-coated PS fibres, and PANI-graphene-coated PS fibres. Inset: derivatives of the TGA degradation curves.

the materials, a total residue of $5 \%$ is left for only the graphenePANI-coated PS fibre.

\subsection{Electrical characterization}

Fig. 11 shows the room-temperature AC electrical conductivity results of the two conducting samples. The whole conductance spectra can be divided into two regions. In case of the PANIcoated PS sample, the low-frequency region shows a very slow and gradual increase in conductivity with the increase in frequency until $10^{6} \mathrm{~Hz}$. Above this frequency, there is an abrupt increase in conductivity. The reasons behind this behaviour of the PANI-PS nanofibre membrane may be the motion of PANI and PS chain dipoles (relaxation region). ${ }^{37}$

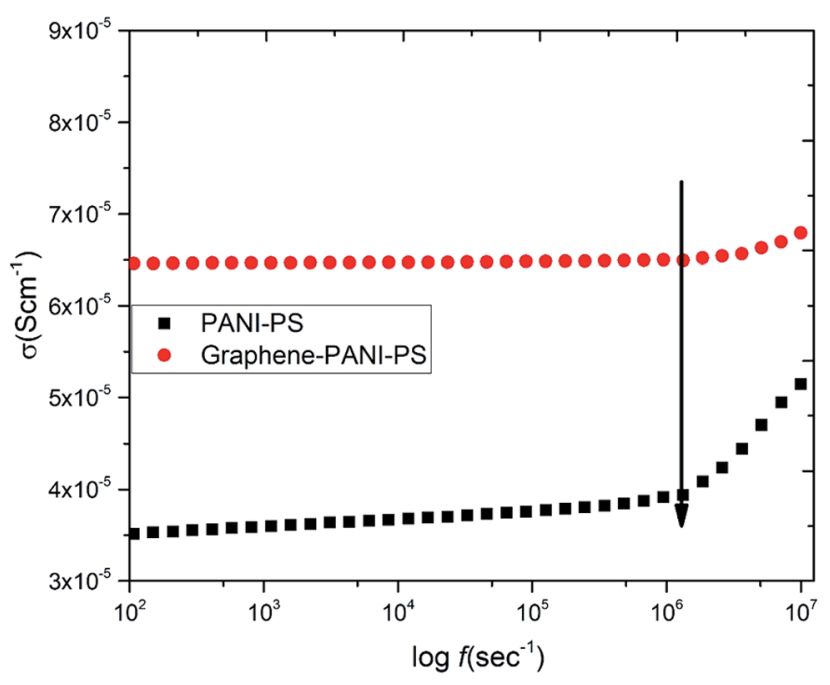

Fig. 11 AC electrical conductivity as a function of frequency for conducting PANI-coated and PANI-graphene-coated PS fibre membranes. 
In the case of the graphene-PANI-PS, the low-frequency region corresponds to a frequency-independent plateau region until $10^{6} \mathrm{~Hz}$. Extrapolation of the plateau to zero frequency gives the value of the DC ionic conductivity. The high-frequency region shows a gradual increase in conductivity. This occurs because the incorporation of graphene along with conducting polymers improves the charge storage capacity of the blends. ${ }^{38}$

\subsection{Sensor measurements}

The gas-sensing ability of the nanocomposite-coated PS membranes is explored. The experimental setup is described in previous work. ${ }^{35}$ The parameters include the sensitivity, response time, selectivity, and repeatability. The summation of these parameters is used to determine the efficiency of the sensors. The sensitivity of a sensor is defined as:

$$
S=\frac{R_{\mathrm{g}}-R_{\mathrm{o}}}{R_{\mathrm{o}}}
$$

where, $R_{\mathrm{g}}$ and $R_{\mathrm{O}}$ are the measured resistances in the presence of an analyte gas and $\mathrm{N}_{2}$, respectively. The response time of a sensor is defined as the total time taken by the sensor to change resistance from $R_{\mathrm{o}}$ to $R_{\mathrm{g}}$ when exposed to an analyte gas and then back to $R_{\mathrm{O}}$ upon exposure to nitrogen.

The ability of a particular sensing material to respond strongly to a specific gas is called the selectivity of the sensor. Another key factor that plays a key role in defining a sensor is its repeatability. The repeatability is defined as the sensing material's capability of repeating similar characteristic behaviours when the same environment is repeated a number of times. This study investigated the selectivity behaviour of both nanocomposite-coated PS fibre membranes using constant concentrations of $\mathrm{CO}_{2}, \mathrm{NH}_{3}$, methanol gas, and ethanol gas at room temperature, as shown in Fig. 12. The bar chart for selectivity shows that both materials show similar trends of behaviours towards all the gases. The best sensing response by both the membrane is observed for $\mathrm{CO}_{2}$ gas. However, graphene-PANI-coated samples have stronger and more prominent sensitivity towards $\mathrm{CO}_{2}$ gas then pure PANI coated membrane. The reason behind that is fact that graphene has

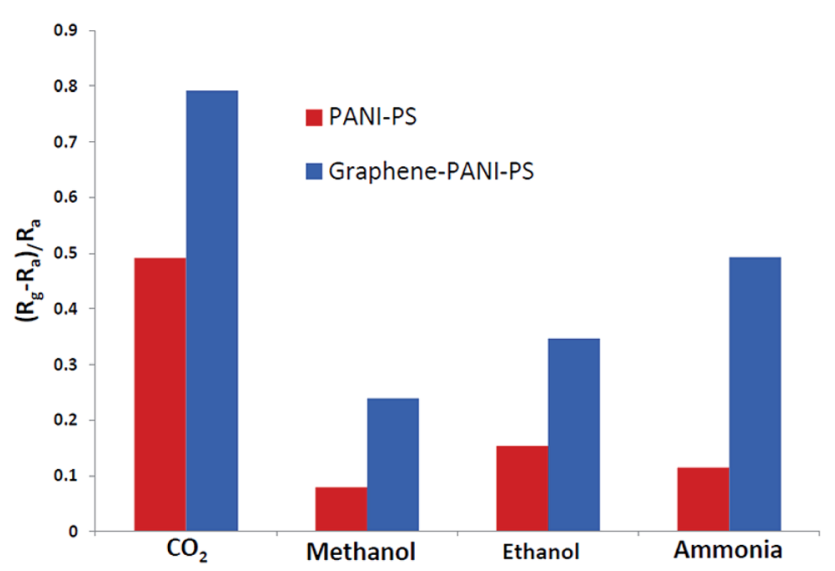

Fig. 12 Selectivity study of conducting membranes when exposed to different analyte gases.

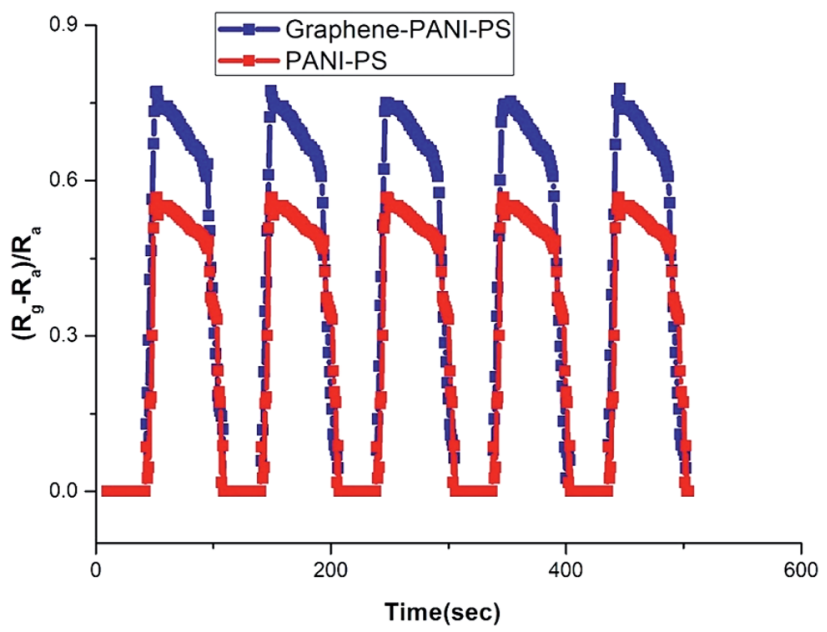

Fig. 13 Repeatability of PANI-coated and graphene-PANI-coated fibre $\mathrm{CO}_{2}$ gas sensors.

a wide electrochemical potential window and has high electron transfer rate. ${ }^{39}$ Therefore, all of the following measurements and investigations are conducted for $\mathrm{CO}_{2}$ gases.

Fig. 13 presents the response time and repeatability of the sensing materials. The response time is defined as the time take by the sensor to show maximum sensation to any particular concentration of analyte gas. Both the samples show same response time with different sensitivity to same concentration of $\mathrm{CO}_{2}$ gas ( $\left.60 \mathrm{ppm}\right)$. The response time for each cycle is almost constant, this indicate the uniform interaction of the sensing material with the gas molecule. The average response time of the sensor is calculated to be 65 seconds, and it is uniform in each cycle. The figure indicates that the sensors show good repeatability.

Fig. 14 reflects change in sensitivity of graphene-PANIcoated sensor to different $\mathrm{CO}_{2}$ concentrations. The graphenePANI-coated sensor showed increase in sensitivity with increase in $\mathrm{CO}_{2}$ concentrations.

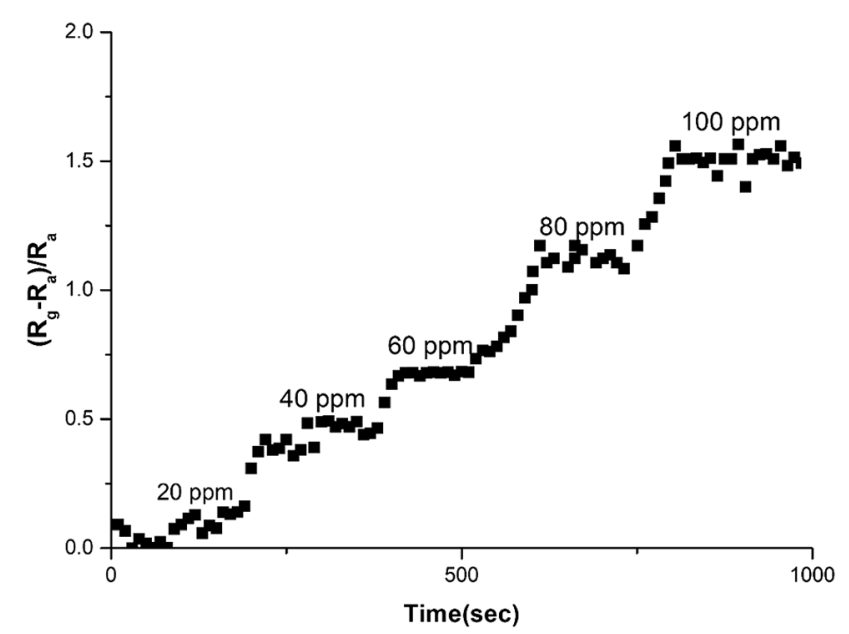

Fig. 14 Sensitivity of graphene-PANI-coated sensor to different $\mathrm{CO}_{2}$ concentrations. 


\section{Conclusion}

PANI-coated and graphene-PANI-coated flexible gas sensors are prepared using an in situ chemical polymerization casting method. The profilometry analysis indicates even distribution of the electrospun fibres in the whole surface layer without any significant differences. Morphological studies using SEM reveals that the diameter of the pure electrospun PS microfibres is $3 \mu \mathrm{m}$, and that of the PANI-nanofibres is $2-10 \mathrm{~nm}$. In addition, PS fibres are well coated with graphene-PANI nanocomposites. The conductive AFM show an increase in the surface roughness and electrical current distribution of the fibres after coating with PANI and PANI-graphene. Moreover, the presence of graphene in the PANI coating resulted in a more even distribution of electrical current in the whole surface area. The presence of chemical interactions between the polymers and nanocomposites is observed in the FTIR results. Clues about the formation of semicrystalline nanocomposites are obtained from the XRD spectra. The thermal stability of the nanocomposite-coated PS fibres is enhanced significantly as observed from thermal studies. The electrical conductivity values are increased slightly by the addition of graphene to the PANI during the nanocomposite formation. Though both nanocomposite-coated gas sensors have the good sensitivity towards $\mathrm{CO}_{2}$ gas, however addition of graphene in nanocomposite shows significant increase in $\mathrm{CO}_{2}$ sensitivity. The response time and recovery time are 65 seconds. Good repeatability is observed when the sensor is exposed to the same concentration of $\mathrm{CO}_{2}$ gas, and the sensitivity increased with increases in the $\mathrm{CO}_{2}$ gas concentration.

\section{Conflicts of interest}

On behalf of all authors, the corresponding author states that there is no conflict of interest.

\section{Acknowledgements}

The authors express their deep sense of gratitude to students of Al-Bairaq for their contribution in the experimental work. Authors would also like to acknowledge the Office of Vice President for Research and Centre for Advanced Materials for the constant support during this research work. Qatar University Student Grant number QUST-2-CAM-2018-4 made this work possible. The authors would like to express their gratitude to the Qatar National Library for financial support to pay open access charges for this publication. The statements made herein are solely the responsibility of the author(s).

\section{References}

1 D. C. D. Bradley and H. Wei, A new flexible venue, npj Flexible Electronics, 2017, 1, 3, DOI: 10.1038/s41528-017-0010-0.

2 T. Wang, Y. Guo, P. Wan, H. Zhang, X. Chen and X. Sun, Flexible Transparent Electronic Gas Sensors, Small, 2016, 12, 3748-3756.
3 https://www.activesustainability.com/environment/effectsair-pollution-human-health/.

4 C. Liu, P. C. Hsu, H. W. Lee, M. Ye, G. Zheng, N. Liu, W. Li and Y. Cui, Transparent air filter for high-efficiency PM2.5 capture, Nat. Commun., 2015, 6, 6205.

5 M. T. Ramesan, V. Santhi, B. K. Bahuleyan and M. A. AlMaghrabi, Structural characterization, material properties and sensor application, study of in situ polymerized polypyrrole/silver doped titanium dioxide nanocomposites, Mater. Chem. Phys., 2018, 211, 343-354.

6 K. C. Persaud, Polymers for chemical sensing, Mater. Today, 2005, 8, 38-44.

7 V. Nair, F. Regius, A. Ragavendar and S. Suman, Role of electrospun ZnO-NiO metaloxide nanocomposite fibers for sensor applications, International Conference on Industrial Engineering and Operations Management (IEOM), 2015, DOI: 10.1109/IEOM.2015.7228112.

8 J. Miao, M. Miyauchi, T. J. Simmons, J. S. Dordick and R. J. Linhardt, Electrospinning of nanomaterials and applications in electronic components and devices, $J$. Nanosci. Nanotechnol., 2010, 10, 5507-5519.

9 (a) Z. Ahmad, Q. Zafar, K. Sulaiman, R. Akram and K. Karimov, A Humidity Sensing Organic-Inorganic Composite for Environmental Monitoring, Sensors, 2013, 13, 3615-3624; (b) I. Olenych, O. Aksimentyeva, Y. Horbenko and B. Tsizh, Organic-inorganic nanocomposites for gas sensing, International Conference Radio Electronics \& Info Communications (UkrMiCo), 2016, DOI: 10.1109/UkrMiCo.2016.7739609.

10 C. Wang, L. Yin, L. Zhang, D. Xiang and R. Gao, Metal Oxide Gas Sensors: Sensitivity and Influencing Factors, Sensors, 2010, 10, 2088-2106.

11 B. Ding, M. Wang, J. Yu and G. Sun, Gas Sensors Based on Electrospun Nanofibers, Sensors, 2009, 9, 1609-1624.

12 A. N. Mallya, R. Kottokkaran and P. C. Ramamurthy, Conducting polymer-carbon black nanocomposite sensor for volatile organic compounds and correlating sensor response by molecular dynamics, Sens. Actuators, B, 2014, 20, 308-320.

13 F. H. Babaei and A. H. Zare, The selective flow of volatile organic compounds in conductive polymer-coated microchannels, Sci. Rep., 2017, 7, 42299.

14 C. Wang, L. Yin, L. Zhang, D. Xiang and R. Gao, Metal Oxide Gas Sensors: Sensitivity and Influencing Factors, Sensors, 2010, 10, 2088-2106, DOI: 10.3390/s100302088.

15 C. Wang, L. Yin, L. Zhang, D. Xiang and R. Gao, Metal Oxide Gas Sensors: Sensitivity and Influencing Factors, Sensors, 2010, 10, 2088-2106.

16 K. Xu, C. Fu, Z. Gao, F. Wei, Y. Ying, C. Xu and G. Fu, Nanomaterial-based gas sensors: a review, Instrum. Sci. Technol., 2018, 46, 115-145.

17 T. Anjitha, T. Anilkumar, G. Mathew and M. T. Ramesan, Zinc Ferrite @ Polyindole Nanocomposites: Synthesis, Characterization and Gas Sensing Applications, Polym. Compos., 2018, DOI: 10.1002/pc.25088.

18 L. Bacakova, E. Filova, J. Liskova, I. Kopova, M. Vandrovcova and J. Havlikova, Nanostructured materials as substrates for 
the adhesion, growth, and osteogenic differentiation of bone cells, Nanobiomaterials in Hard Tissue Engineering, ed. Alexandru Mihai Grumezescu, William Andrew Publishing, 2016, ch. 4, pp. 103-153.

19 J. J. Ramsden, Nanomaterials and their Production, in Micro and Nano Technologies, Nanotechnology, ed. Jeremy J. Ramsden, William Andrew Publishing, 2011, ch. 6, pp. 101-124.

20 S. Iravani, H. Korbekandi, S. V. Mirmohammadi and B. Zolfaghari, Synthesis of silver nanoparticles: chemical, physical and biological methods, Res. Pharm. Sci., 2014, 9, 385-406.

21 M. E. Grigore, E. R. Biscu, A. M. Holban, M. C. Gestal and A. M. Grumezescu, Methods of Synthesis, Properties and Biomedical Applications of $\mathrm{CuO}$ Nanoparticles, Pharmaceuticals, 2016, 9, 75.

22 P. Lu and B. Ding, Applications of electrospun fibers, Recent Pat. Nanotechnol., 2008, 2, 169-182.

23 A. Haynes and P. I. Gouma, Polyaniline-Based Environmental Gas Sensors, in Sensors for Environment, Health and Security, ed. M. I. Baraton, NATO Science for Peace and Security Series C: Environmental Security, Springer, Dordrecht, 2009, pp. 451-459 .

24 J. Y. Shimano and A. G. MacDiarmid, Polyaniline, a dynamic block copolymer: key to attaining its intrinsic conductivity?, Synth. Met., 2001, 123, 251-262.

25 W. Lyu, M. Yu, J. Feng and W. Yan, Highly crystalline polyaniline nanofibers coating with low-cost biomass for easy separation and high efficient removal of anionic dye ARG from aqueous solution, Appl. Surf. Sci., 2018, 458, 413-424.

26 S. W. Ng, N. Noor and Z. Zheng, Graphene-based twodimensional Janus materials, NPG Asia Mater., 2018, 10, 217-237.

27 V. Singh, D. Joung, L. Zhai, S. Das, S. I. Khondaker and S. Seal, Graphene based materials: past, present and future, Prog. Mater. Sci., 2011, 56, 1178-1271.

28 M. I. Katsnelson, Graphene: carbon in two dimensions, Mater. Today, 2007, 10, 20-27.

29 S. Basu and P. Bhattacharyya, Recent developments on graphene and graphene oxide based solid state gas sensors, Sens. Actuators, B, 2001, 173, 1-21.
30 U. Latif and F. L. Dickert, Graphene Hybrid Materials in Gas Sensing Applications, Sensors, 2015, 5, 30504-30524.

31 Y. Li, A. Y. Samad, K. Polychronopoulou, S. M. Alhassan and K. Liao, Highly Electrically Conductive Nanocomposites Based on Polymer Infused Graphene Sponges, Sci. Rep., 2018, 39, 3858-3868.

32 A. Kumar, V. Kumar, M. Kumar and K. Awasthi, Synthesis and characterization of hybrid PANI/MWCNT nanocomposites for EMI applications, Polym. Compos., 2017, DOI: $10.1002 / \mathrm{pc} .24418$.

33 U. Male, P. Srinivasan and B. S. Singu, Incorporation of polyaniline nanofibres on graphene oxide by interfacial polymerization pathway for supercapacitor, Int. Nano Lett., 2015, 5, 231-240.

34 M. T. Ramesan and T. Sampreeth, Synthesis, characterization, material properties and sensor application study of polyaniline/niobium doped titanium dioxide nanocomposites, J. Mater. Sci.: Mater. Electron., 2017, 28, 16181-16191.

35 J. Bhadra, N. J. Al-Thani, N. K. Madi and M. A. Al-Maadeed, Preparation and characterization of chemically synthesized polyaniline-polystyrene blends as a carbon dioxide gas sensor, Synth. Met., 2013, 181, 27-36.

36 M. T. Ramesan and V. Santhi Synthesis, characterization, conductivity and sensor application study of polypyrrole/ silver doped nickel oxide nanocomposites, Compos. Interfaces, 2018, 25(8), 725-741.

37 S. Saravanan, M. R. Anantharaman and S. Venkatachalam, Structural and electrical studies on tetrameric cobalt phthalocyanine and polyaniline composites, Mater. Sci. Eng. C, 2006, 135, 113-119.

38 J. Bhadra, N. J. Al-Thani, N. K. Madi and M. A. Al-Maadeed, High performance sulfonic acid doped polyanilinepolystyrene blend ammonia gas sensors, J. Mater. Sci.: Mater. Electron., 2016, 27, 8206-8216.

39 K. I. Bolotin, K. J. Sikes, Z. Jiang, M. Klima, G. Fudenberg, J. Hone, P. Kim and H. L. Stormer, Ultrahigh electron mobility in suspended graphene, Solid State Commun., 2008, 146, 351-355. 